\title{
FONTE DE INFORMAÇÃO SOBRE OS BENEFÍCIOS DA ATIVIDADE FÍSICA E SUA RELAÇÃO COM A PRÁTICA EFETIVA EM ADOLES- CENTES DA ZONA RURAL
}

\author{
Marcelo Cozzensa da Silva \\ Universidade Federal de Pelotas, Pelotas, Rio Grande do Sul, Brasil \\ Rui Carlos Gomes Dorneles \\ Universidade Federal de Pelotas, Pelotas, Rio Grande do Sul, Brasil \\ Eduardo Lucia Caputo \\ Universidade Federal de Pelotas, Pelotas, Rio Grande do Sul, Brasil
}

\begin{abstract}
Resumo
O objetivo do estudo foi verificar quais as fontes de informação utilizadas por estudantes do Ensino Médio da zona rural sobre os benefícios da prática de atividade física (AF) e sua relação com a prática efetiva. Foi realizado um estudo transversal de base escolar com 510 adolescentes, no qual se avaliaram aspectos demográficos, socioeconômicos, comportamentais, nutricionais, de saúde e as fontes de informação sobre os benefícios da atividade física para a saúde. Médico, parente/amigo e meios de comunicação demonstraram-se associados à prática de AF. É importante que sejam realizadas intervenções com os alunos, assim como no ambiente familiar, em razão de a maior relação entre fonte de informação e prática efetiva de atividades físicas se estabelecer nesses domínios.
\end{abstract}

Palavras-chave: Informação. Atividade física. Adolescente. Zona rural.

\section{Introdução}

A inatividade física pode ser considerada um dos grandes males das últimas décadas, sendo associada a maiores riscos de algumas morbidades, tais como doenças coronárias, diabetes tipo 2 e câncer de mama e cólon (LEE et al., 2012). Em contrapartida, a prática regular de atividades físicas, em razão de seus benefícios à saúde, é uma das principais formas de prevenção e tratamento de doenças na área de saúde pública (OMS, 2010).

A manutenção e o aprimoramento da prática de atividade física durante a adolescência apresentam importante contribuição para a saúde dos indivíduos nessa faixa etária (KOPCAKOVA et al., 2015). Tais benefícios encontram-se tanto em aspectos físicos quanto em psicossociais e cognitivos (EIME et al., 2013). Controle de peso corporal, melhora nas relações interpessoais, aumento da aptidão física, entre outros, são alguns dos ganhos obtidos por essa população ao serem ativos fisicamente (CAGLAR; CANLAN; DEMIR, 2009; KWON; LEE; CARNETHON, 2015). 
Tais benefícios descritos anteriormente, além de outras informações relativas à saúde, são rastreados pelos jovens através de diversas meios, sendo a televisão, a internet, amigos, livros, revistas, jornais, médico e família os mais frequentes (MARTINS et al., 2008). Entretanto, a busca por informação parte das necessidades, motivações e competências do indivíduo. Por exemplo, questões relacionadas à sexualidade e a doenças sexualmente transmissíveis estão entre as mais procuradas pelos jovens, tendo em vista que, nesse período da vida, tal assunto se torna expressivo em seu cotidiano (ROMERO et al., 2007; OLIVEIRA et al., 2009).

Apesar de programas de intervenção com atividade física terem encontrado melhores efeitos ao envolver a família na prática do jovem (VAN SLUIJS; MCMINN; GRIFFIN, 2008), não se sabe quais fontes de informação essa população utiliza para se informar sobre tal tema. Além disso, populações residentes nos meios rural e urbano têm estilos de vida bastante distintos. Mesmo quando pertencentes a uma mesma região geográfica, apresentam hábitos comportamentais diferenciados (GLANER, 2005) e, quando semelhantes, podem ser determinados por diferentes preditores. Dentro deste contexto, o objetivo do presente estudo foi verificar quais são as fontes de informação utilizadas por estudantes do Ensino Médio da zona rural da região sul do Rio Grande do Sul sobre os benefícios da prática regular de AF e sua relação com a prática efetiva.

\section{Metodologia}

O estudo caracteriza-se como sendo do tipo observacional de corte transversal. A metodologia completa encontra-se no artigo de Barros e Silva (2013). Todos os alunos pertencentes ao $1^{\circ}, 2^{\circ}$ e $3^{\circ}$ anos das escolas de Ensino Médio da zona rural foram convidados a participar do estudo. Foram recrutados estudantes com idade entre 13 e 19 anos. Entre as escolas estudadas, quatro estavam localizadas no município de Canguçu, duas em São Lourenço do Sul, duas em Pelotas, uma em Piratini e uma em Cerrito.

Foi realizado contato preliminar com a escola, a fim de verificar o interesse em participar do estudo. Foram realizadas duas visitas: a primeira, com o objetivo de explicitar os objetivos do estudo e entregar o termo de consentimento; e a segunda, para a aplicação do questionário. Para a coleta de dados, foi utilizado um questionário contendo questões demográficas (sexo: masculino, feminino; idade: anos completos; cor da pele: branco, não branco); socioeconômicas (escolaridade: $1^{\circ}$ ano, $2^{\circ}$ ano, $3^{\circ}$ ano do ensino médio), comportamentais (hábito de fumar: fumou nos últimos 30 dias: não, sim; consumo de bebidas alcoólicas nos últimos 30 dias: não, sim; nível de atividade física: insuficientemente ativo, ativo) e antropométricas (peso: em quilogramas e estatura: em centímetros). A mensuração do peso e da estatura foi realizada por medida direta, respectivamente, por meio de uma balança da marca Slim Control, com capacidade de $150 \mathrm{~kg}$ e com precisão de $100 \mathrm{~g}$, e fita métrica metálica inextensível, tamanho de $150 \mathrm{~cm}$ e com precisão de $1,0 \mathrm{~mm}$.

A fonte de informação sobre benefícios da atividade física foi coletada em duas etapas: a primeira, através da questão "Alguém já lhe informou que seria bom fazer exercícios físicos para melhorar sua saúde?". Em caso positivo, era solicitado ao adolescente marcar a(s) opção(ões) (múltipla escolha) de onde foi oriunda a informação: médico, parente/amigo, professor, meio de comunicação (televisão, rádio, revista, jornal). O desfecho, prática de atividade física no lazer, foi coletado com base no instrumento elaborado por Farias Júnior et al. (2012), o qual mede (sem contar as práticas executadas durante a disciplina de educação física escolar) as atividades físicas realizadas no tempo livre, o número de dias e horas por dia praticadas. Foram definidos como insuficientemente ativos no lazer os adolescentes que realizaram menos de 300 minutos de atividade física moderada a vigorosa por semana (BIDDLE; SALLIS; CAVILL, 1998). 
Os dados do questionário foram duplamente digitados utilizando-se o programa Epi Info 6.0, com checagem automática de consistência e também checagem manual para correção de possíveis erros. A análise descritiva caracterizou a amostra total, conforme variáveis demográficas, socioeconômica, comportamentais, nutricional e em relação a ter recebido informação sobre os benefícios da atividade física. Na análise bruta, a associação entre as variáveis independentes e o desfecho (nível de atividade física na forma categórica) foi realizada por meio de Regressão de Poisson. A análise dos dados foi realizada no programa estatístico Stata 12.0. O projeto foi submetido e aprovado pelo Comitê de Ética em Pesquisa da Faculdade de Educação Física da Universidade Federal de Pelotas (Protocolo nº 038/2011).

\section{Resultados}

Foram entrevistados 510 adolescentes, com média de idade de 16,3 anos ( $\mathrm{DP}=1,2$ anos). A maioria dos entrevistados se encontrava na faixa etária de 16 anos $(27,7 \%)$, era do sexo feminino (59\%) e com cor de pele branca $(92,3 \%)$. Quanto à escolaridade, $37,7 \%$ dos adolescentes estavam cursando o primeiro ano do Ensino Médio.

Com relação às variáveis comportamentais, 69,9\% dos entrevistados tinham ingerido bebidas alcoólicas e pouco mais da metade $(53,3 \%)$ praticava a quantidade mínima de 300 minutos de atividade física semanais recomendada para benefícios à saúde. Com relação ao desfecho principal do estudo (fontes de informação sobre benefícios da atividade física), 90,4\% dos participantes receberam alguma informação sobre os benefícios da atividade física para a saúde (Tabela 1).

Tabela 1. Descrição das variáveis demográficas, antropométricas e comportamentais de escolares pertencentes à $5^{\mathrm{a}}$ CRE-RS do Ensino Médio da zona rural, 2012.

\begin{tabular}{lcc}
\hline Variáveis & $\underline{\mathbf{n}}$ & $\underline{(\%)}$ \\
\hline $\begin{array}{l}\text { Sexo }(N=510) \\
\quad \text { Masculino }\end{array}$ & 209 & $(41,0)$ \\
$\quad$ Feminino & 301 & $(59,0)$ \\
Idade $(N=510)$ & & \\
$\quad 13 / 14$ anos & 25 & $(4,9)$ \\
$\quad 15$ anos & 131 & $(25,7)$ \\
16 anos & 141 & $(27,7)$ \\
17 anos & 125 & $(24,5)$ \\
$18 / 19$ anos & 88 & $(17,2)$ \\
Cor da pele $(N=492)$ & & \\
$\quad$ Branca & 454 & $(92,3)$ \\
$\quad$ Não branca & 38 & $(7,7)$ \\
Escolaridade $(N=510)$ & & \\
$\quad 1^{\circ}$ ano & 192 & $(37,7)$ \\
$2^{\circ}$ ano & 154 & $(30,1)$ \\
$3^{\circ}$ ano & 164 & $(32,2)$ \\
Tabagismo nos últimos 30 dias $(N=502)$ & & \\
$\quad$ Não & 465 & $(92,6)$ \\
Sim & 37 & $(7,4)$ \\
Consumo de álcool nos últimos 30 dias $(N=505)$ & & \\
$\quad$ Não & 167 & $(33,1)$ \\
Sim & 338 & $(69,9)$ \\
Nível de atividade física $(N=510)$ & &
\end{tabular}


$\begin{array}{ll}\text { Insuficientemente ativo } & 238\end{array}$

Ativo

$\operatorname{IMC}(N=509)$

Normal

Sobrepeso

422

Obesidade

Recebeu informação sobre benefício da AF para saúde

$(N=509)$

Não

Sim

Fonte: Os autores, 2012

Na Figura 1, são apresentados os dados em relação à fonte de informação recebida sobre os benefícios da prática de atividades físicas à saúde. Foi verificado que $65 \%$ dos adolescentes receberam informações advindas de parentes e amigos; 38\%, por meio de médicos; $76 \%$, de professores de qualquer disciplina, e $78 \%$, através dos meios de comunicação.

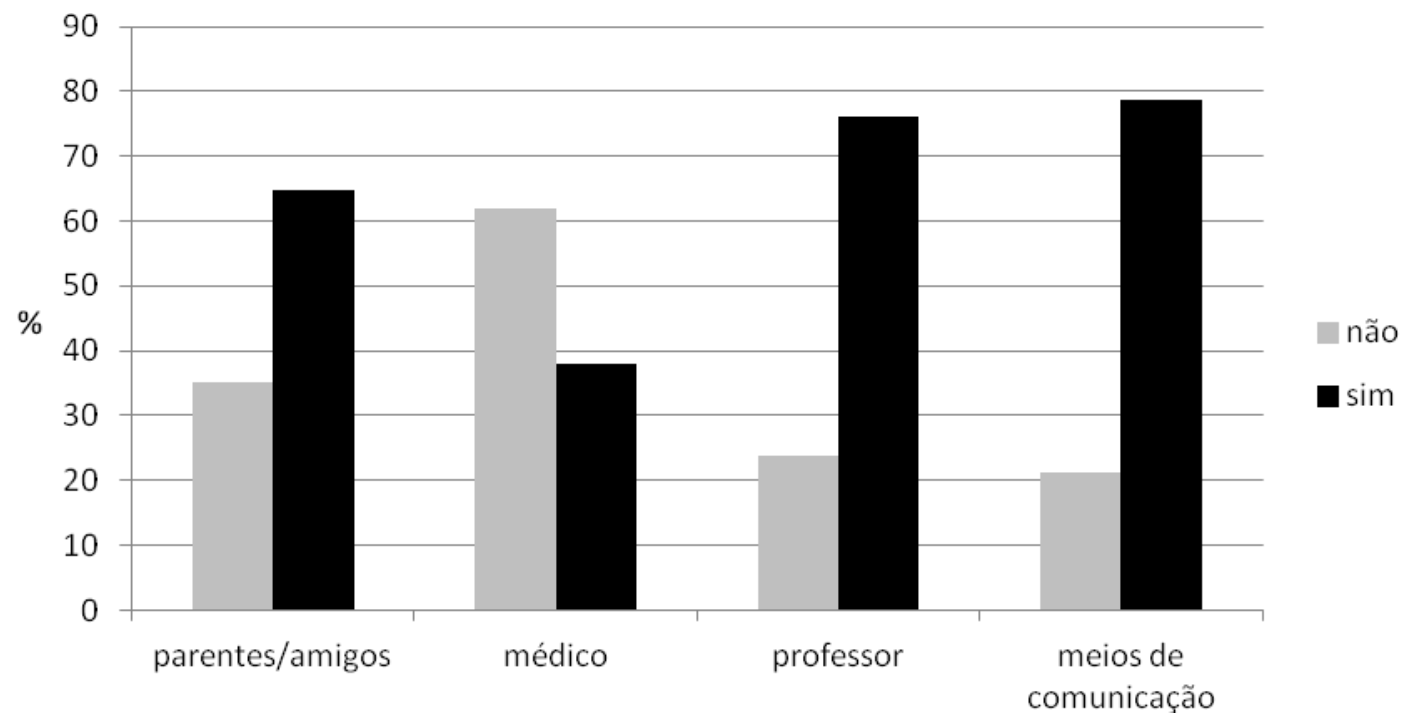

Figura 1. Frequência das fontes de informação recebidas sobre os benefícios da prática de atividades físicas à saúde $(n=460)$.

Fonte: Os autores, 2012

A análise bruta e ajustada sobre a relação entre as fontes de informação sobre os benefícios da prática de atividade física e a prática efetiva de AF está demonstrada na Tabela 2. Na análise bruta, observou-se uma associação significativa entre todas as fontes de informação e a prática efetiva de AF. Na análise ajustada (controlada para as variáveis sexo, cor de pele, idade, renda familiar, tabagismo, consumo de álcool e IMC), observou-se que as fontes de informação médico $(p=0,04)$, parente/amigo $(p=0,001)$ e meios de comunicação $(p=0,01)$ permaneceram significativamente associadas ao desfecho. Adolescentes que receberam informação da fonte parente/amigo obtiveram $43 \%$ mais chance de prática suficiente de AF quando comparados àqueles que não receberam informação desta fonte. 
Tabela 2. Análise bruta e ajustada de prática suficiente de atividade física e fontes de informação de benefícios da prática à saúde entre escolares pertencentes à $5^{\text {a }}$ CRE-RS do Ensino Médio da zona rural, 2012.

\begin{tabular}{lcccc}
$\begin{array}{l}\text { Fonte de informa- } \\
\text { ção }\end{array}$ & $\underline{\text { Bruto }}$ & \multicolumn{1}{c}{ Ajustado* } \\
Médico & $\underline{\mathrm{RP}(\mathrm{IC} 95 \%)}$ & $\mathrm{p}^{\#}$ & $\underline{\mathrm{RP}(\mathrm{IC} 95 \%)}$ & $\mathrm{p}^{\#}$ \\
$\begin{array}{l}\text { Parente/amigo } \\
\text { Professor }\end{array}$ & $1,48(1,03-1,46)$ & 0,02 & $1,19(1,01-1,41)$ & 0,04 \\
$\begin{array}{l}\text { Meios de comuni- } \\
\text { cação }\end{array}$ & $1,27(1,01-1,63)$ & $<0,001$ & $1,43(1,16-1,75)$ & 0,001 \\
& $1,50(1,14-1,96)$ & 0,04 & $1,14(0,91-1,42)$ & 0,25 \\
\end{tabular}

*Ajustado para sexo, cor da pele, idade, renda familiar, tabagismo, consumo de álcool e índice de massa corporal; $\mathrm{p}^{\#}$ : valor $\mathrm{p}$, teste de Walt para heterogeneidade; IC95\%: intervalo de confiança de 95\%; RP: razão de prevalência.

Fonte: Os autores, 2012

\section{Discussão}

Os dados do presente estudo indicaram que mais de $90 \%$ dos adolescentes já receberam alguma informação sobre os benefícios da atividade física para a saúde e, destes, a maioria $(78 \%)$ obteve tais informações através de meios de comunicação. Ainda, aqueles que receberam informações provenientes de médicos, parentes e amigos e meios de comunicação demonstraram maior relação com a prática efetiva comparados àqueles que não receberam tais informações destas fontes.

Mais da metade dos entrevistados foi considerada fisicamente ativo, valor superior ao encontrado, nos estudos de Glaner (2002), com adolescentes da zona rural (41,7\%) e urbana $(24,0 \%)$ e de Silveira e Silva (2011), realizado com adolescentes moradores na zona urbana da mesma região (36\%). Segundo Glanner (2002), um dos principais motivos dessa diferença é que adolescentes residentes em zonas rurais têm maior nível de atividade física em seus hábitos diários, como trabalho e deslocamento, diferentemente de adolescentes residentes em área urbana, os quais concentram suas atividades físicas somente no lazer. Além disso, segundo Silva et al. (2009), atualmente, o tempo de esforço físico dispendido no lazer entre adolescentes é muito baixo.

A frequência de estudantes da zona rural que já havia recebido informações sobre benefícios da prática regular de AF foi de $90 \%$. Adolescentes que frequentam uma ou mais atividades relacionadas à saúde no ambiente escolar revelaram, em média, uma autopercepção positiva do impacto destas informações (GASPAR, 2006). Dunton e Schneider (2006) e Coll, Amorim e Hallal (2010) indicam que informações sobre a prática regular de AF vêm sendo amplamente abordadas e debatidas tanto entre a comunidade científica como nos meios de comunicação, o que ajuda a explicar a prevalência encontrada.

Outro achado do presente estudo diz respeito à maioria dos alunos afirmarem que as principais fontes de informações vieram dos meios de comunicação, professores e parentes, respectivamente, sendo através dos médicos a menor parcela de aconselhamento. O estudo de Booth et al. (1997) explica em parte tal achado ao constatar que a prevalência do médico como fonte preferida de informação cresce conforme o aumento da idade. Em seu estudo, mais de $50 \%$ dos indivíduos com 60 ou mais anos de idade declararam o médico como principal fonte de suporte para benefícios da AF para saúde, ao passo que somente $22 \%$ dos jovens declararam tal fonte. Estudo de Mendes et al. (2010) descreve a importância dos meios de comunicação como fonte de informação sobre a importância da AF devido à sua natureza abrangente. Apesar disso, questionam a capacidade das informações advindas dessa fonte de 
mudar hábitos de vida dos indivíduos para que efetivamente estes sigam as orientações recomendadas pelos órgãos de promoção de saúde.

Alguns estudos descrevem que a família é uma das maiores fontes de informação de saúde para os adolescentes, atuando como um fator protetor para comportamentos de risco por parte destes indivíduos. Da mesma forma, a escola é um espaço social significativo para o adolescente, pois é o local onde este compartilha suas experiências, curiosidades e inquietações (ROMERO et al., 2007; OLIVEIRA et al., 2009). É, contudo, necessária a capacitação dos profissionais que atuam nesse ambiente para que possam intervir de forma adequada, promovendo discussões e reflexões entre esses jovens (BORGES; NICHIATA; SCHOR, 2006).

Ao avaliarmos a relação entre cada uma das fontes de informação sobre os benefícios da prática de atividade física com a prática efetiva, constatou-se que as informações oriundas de médicos, parentes/amigos e meios de comunicação estiveram associadas à prática desses adolescentes. Ainda que a segunda maior frequência de fonte de informações sobre os benefícios da AF seja através dos professores, esta foi a única que não apresentou associação significativa com a prática efetiva. Indivíduos que recebem aconselhamentos de profissionais da saúde apresentam maiores chances de mudança de hábitos (CALFAS et al., 1997). Ao revisar a literatura, encontraram-se estudos sobre fontes de informação de adolescentes relativos a sexualidade (ROMERO et al., 2007), HIV/AIDS (CAMARGO; BOTELHO, 2007) e gravidez na adolescência (MOURA et al., 2011). A falta de estudos referentes ao desfecho estudado na presente pesquisa demonstra a importância e a necessidade de se aprofundar na referida temática. Deve-se levar em consideração que esses adolescentes revelaram, efetivamente, praticar atividade física quando a informação sobre os seus benefícios foi oriunda de parentes/amigos e de médicos. Entretanto, a escola, por sua natureza plenamente formativa e informativa, não deve ser deixada de lado, pois nela existe o potencial de desenvolvimento de projetos e trabalhos cooperativos de profissionais da saúde atuando dentro do contexto escolar. Desta forma, procurar-se-iam aproximar os canais de informação para um maior alcance dos indivíduos, buscando atingir maiores níveis de prática efetiva.

Deve-se enfatizar que este é um dos primeiros estudos a verificar a relação entre prática e fonte de informação sobre atividade física entre adolescentes da zona rural no Brasil. Entretanto, algumas limitações devem ser descritas, como o delineamento utilizado (transversal), o qual nos impossibilita identificar causalidade entre os acontecimentos, e a não existência de um questionário validado para a coleta de informações sobre as fontes de informação.

Concluindo, os achados indicaram que pouco mais da metade dos adolescentes rurais foram considerados suficientemente ativos e $90 \%$ dos alunos das escolas estudadas relataram ter recebido informações sobre a importância da prática regular de atividades físicas. Destes, a maioria recebeu essas informações através dos meios de comunicação e de professores de todas as disciplinas. Todavia, apesar de receberem grande quantidade de informação dos professores, tal fonte de informação não teve relação significativa com a prática efetiva. Quando essas informações são recebidas de parentes e amigos, meios de comunicação e médicos, os resultados mostraram associação entre a informação recebida e a prática efetiva, independente de sexo, cor de pele, idade, renda familiar, tabagismo, consumo de álcool e índice de massa corporal, quando comparados a quem não recebe informação dessa população.

\section{Conclusões}

Com base nos achados, percebe-se que são necessárias intervenções não somente entre os alunos, mas também no ambiente familiar (pais e amigos), em razão de a maior relação entre fonte de informação e prática efetiva de atividades físicas se estabelecer nesses domínios. Além disso, qualificar os diferentes profissionais da área da saúde, em especial os médi- 
cos, pode ajudar a fortalecer e ampliar as informações aos adolescentes. Ainda é necessário identificar a razão pela qual a figura do professor de Educação Física não impacta como fonte de modificação dos hábitos de prática destes adolescentes do meio rural.

\title{
SOURCE OF INFORMATION ABOUT THE BENEFITS OF PHYSICAL ACTIVITY AND ITS RELATION TO TEENS EFFECTIVE PRACTICE IN RURAL AREA
}

\begin{abstract}
The study objective was to verify that the information sources used by high school students from rural areas about the benefits of physical activity (PA) and its relationship to actual practice. A cross-sectional school-based with 510 adolescents which evaluated aspects demographic, socioeconomic, behavioral, nutritional, health, and sources of information about the benefits of physical activity to health was conducted. Doctor, relative / friend and media proved to be associated with PA practice. It is important that interventions with students, as well as in the family environment due to greater relationship between source of information and the effective physical activity to settle in these areas.

Keywords: Information. Physical activity. Adolescent. Rural areas.

\section{FUENTE DE INFORMACIÓN SOBRE LOS BENEFICIOS DE LA ACTIVIDAD FÍ- SICA Y SU RELACIÓN CON LA PRÁCTICA EFECTIVA EN ADOLESCENTES DE LA ZONA RURAL}

\section{Resumen}

El objetivo del estudio fue verificar cuáles son las fuentes de información utilizadas por los estudiantes de secundaria de las zonas rurales sobre los beneficios de la actividad física (AF) y, cuál es su relación con la práctica real. Se desarrolló un estudio transversal de base escolar con 510 adolescentes. En ese estudio se evaluaron aspectos demográficos, socioeconómicos, comportamentales, nutricionales, de salud y las fuentes de información sobre los beneficios de la actividad física para la salud. Médico, pariente/ amigo y medios de comunicación demostraron estar asociados con la práctica de AF. Es importante que se realicen intervenciones con los estudiantes e, igualmente, en el ambiente familiar, debido a que la mayor relación entre la fuente de información y la práctica efectiva de las actividades físicas se establece en esos dominios.

Palabras clave: Información. Actividad física. Adolescente. Medio rural.

\section{Referências}

BARROS, F. C.; SILVA, M. C. Conhecimento sobre atividade física e fatores associados em adolescentes estudantes do ensino médio da zona rural. Revista Brasileira de Atividade Física e Saúde, v.18, n. 5, p. 594-603, set. 2013.

BIDDLE, S.; SALLIS, J. F.; CAVILL, N. A. Young and active? Young people and health enhancing physical activity. Evidence and implication. London: Health Education Authority, 1998.

BOOTH, M. L.; BAUMAN, A.; OWEN, N.; GORE, C. J. Physical activity preferences, preferred sources of assistance, and perceived barriers to increased activity among physically inactive Australians. Preventive Medicine, v. 26, n. 1, p. 131-137, jan. 1997. 
BORGES, A. L. V.; NICHIATA, L. Y. I,; SCHOR, N. Conversando sobre sexo: a rede sociofamiliar como base de promoção da saúde sexual e reprodutiva de adolescentes. Revista Latino-americana Enfermagem, v.14, n. 3, p. 422-427, maio/jun. 2006.

CAGLAR, E.; CANLAN, Y.; DEMIR, M. Recreational exercise motives of adolescents and young adults. Journal of Human Kinetics, v. 22, p. 83-89, 2009.

CALFAS, K. J.; SALLIS J.F.; OLDENBURG, B.; FFRENCH, M. Mediators of change in physical activity following an intervention in primary care: PACE. Preventive Medicine, v. 26, n. 3, p. 297-304, maio 1997.

CAMARGO, B. V.; BOTELHO, L. J. Aids, sexualidade e atitudes de adolescentes sobre proteção contra o HIV. Revista de Saúde Pública, v. 41, n. 1, fev. 2007.

COLL, C.; AMORIM, T.E.C.; HALLAL, P.C. Percepção de adolescentes e adultos referente à influência da mídia sobre o estilo de vida. Revista Brasileira de Atividade Física e Saúde, v.15, n. 2, p.105-110, 2010.

DUNTON, G. F.; SCHNEIDER, M. Perceived barriers to walking for physical activity. Preventing Chronic Disease, v. 3, n. 4, p. A116, out. 2006.

EIME, R. M.; YOUNG, J.A.; HARVEY, J.T.; CHARITY, M.J.; WARREN R PAYNE, W.RA systematic review of the psychological and social benefits of participation in sport for children and adolescents: informing development of a conceptual model of health through sport. International Journal of Behavioral Nutrition and Physical Activity, v. 10, n. 98, p. $1,2013$.

FARIAS JÚNIOR, J. C., et al. Validade e reprodutibilidade de um questionário para medida de atividade física em adolescentes: uma adaptação do Self-Administered Physical Activity Checklist. Revista Brasileira de Epidemiologia, v.15, n.1, p.198-210, mar. 2012.

GASPAR, P. J. S. Educação para saúde e estudantes: as fontes e o impacto da informação. 2006. 236 f. Dissertação (Mestrado em Comunicação e Educação em Ciência) - Escola Superior de Saúde, Departamento de Ciências da Educação, Universidade do Aveiro, Aveiro, 2006.

GLANER, M. F. Aptidão física relacionada à saúde de adolescentes rurais e urbanos em relação a critérios de referência. Revista Brasileira de Educação Física e Esporte, v.19, n.1, p.13-24, 2005.

GLANER, M. F. Nível de atividade física e aptidão física relacionada à saúde em rapazes rurais e urbanos. Revista Paulista de Educação Física, v.16, n.1, p.76-85, jan./jun. 2002.

LEE, I. M.; SHIROMA, E.J.; LOBELO, F.; PUSKA, P.; BLAIR, S.N.; KATZMARZYK, P.T. Effect of physical inactivity on major non-communicable diseases worldwide: an analysis of burden of disease and life expectancy. The Lancet, v. 380, n. 9838, p. 219-229, jul. 2012. 
KOPCAKOVA, J.; VESELSKA, Z.D.; GECKOVA, A. M.; KALMAN, M.; VAN DIJK, J. P.; REIJNEVELD, S.A. Do motives to undertake physical activity relate to physical activity in adolescent boys and girls? International Journal of Environmental Research and Public Health, v. 12, n. 7, p. 7656-7666, jul. 2015.

KWON, S.; LEE, J.; CARNETHON, M. R. Developmental trajectories of physical activity and television viewing during adolescence among girls: National Growth and Health Cohort Study. BMC Public Health, v. 15, n. 1, jul. 2015.

MARTINS, L. N. B., et al. Informação sobre contracepção e sexualidade entre adolescentes que vivenciaram uma gravidez. Acta Paulista de Enfermagem, v. 24, n. 3, 320-326, 2011.

MENDES, M. A. et al. Fontes de informação sobre a importância da atividade física: estudo de base populacional. Revista Brasileira de Atividade Física e Saúde, v. 15, n. 3, p. 163$169,2010$.

MOURA, L. N. B., et al. Informação sobre contracepção e sexualidade entre adolescentes que vivenciaram uma gravidez. Acta Paulista de Enfermagem, v. 24, n. 3, p. 320-326, 2011.

OLIVEIRA, D. C.; PONTES, A. P. M.; GOMES, A. M. T.; RIBEIRO, M. C. M. Conhecimentos e práticas de adolescentes acerca das DST/HIV/AIDS em duas escolas públicas municipais do Rio de Janeiro. Escola Anna Nery Revista Enfermagem, v.13, n. 4, p. 833-841, out./dez. 2009.

ROMERO, K. T., et al. O conhecimento das adolescentes sobre questões relacionadas ao sexo. Revista da Associação Médica Brasileira, v. 53, n.1, p.14-19 jan/fev. 2007.

SILVA, D. A. S., et al. Nível de atividade física e comportamento sedentário em escolares. Revista Brasileira de Cineantropometria e Desempenho Humano, v.11, n.3, p. 299-306, 2009.

SILVEIRA, E. F.; SILVA, M. C. Conhecimentos sobre atividade física em estudantes de uma cidade do sul do Brasil. Motriz, v.17, n. 3, p. 456-467, jul/set. 2011.

VAN SLUIJS, E. M.; MCMINN, A. M.; GRIFFIN, S. J. Effectiveness of interventions to promote physical activity in children and adolescents: systematic review of controlled trials. British Journal of Sports Medicine, v. 42, n. 3, p. 653-657, ago. 2008.

TELAMA, R. Tracking of physical activity from childhood to adulthood: a review. Obesity Facts, v. 2, n. 3, p.187-195, jul. 2009.

Recebido em: 07/03/2016

Revisado em: 08/11/2016

Aprovado em: 05/09/2017

Endereço para correspondência:

duducaputo@ hotmail.com

Eduardo Lucia Caputo

Universidade Federal de Pelotas

Rua Gomes Carneiro, 1, Centro, Pelotas - RS, 96010-610 